\title{
NOMA-based D2D Communications: Towards 5G
}

\author{
Jingjing Zhao*, Yuanwei Liu*, Kok Keong Chai*, Yue Chen*, Maged Elkashlan*, and Jesus Alonso-Zarate ${ }^{\dagger}$ \\ * Queen Mary University of London, London, UK \\ $\dagger$ Centre Tecnològic de Telecomunicacions de Catalunya (CTTC), Barcelona, Spain
}

\begin{abstract}
In this paper, a novel non-orthogonal multiple access (NOMA)-based device-to-device (D2D) communications framework is proposed. A major novelty of the proposed framework is that it introduces the new concept of "D2D group" which utilizes NOMA transmission, enabling one D2D transmitter to communicate with multiple D2D receivers simultaneously. Based on the considered framework, a resource allocation optimization problem is formulated, where multiple D2D groups are allowed to reuse the same subchannel. The objective of this work is to maximize the system sum rate by satisfying the signalto-interference-plus-noise (SINR) constraints of both D2D and traditional cellular users. Note that the formulated problem is non-deterministic polynomial-time (NP) hard in nature, thus a novel resource allocation algorithm based on the many-to-one twosided matching theory is proposed for obtaining a suboptimal solution. It is proved that the proposed algorithm converges to a stable state within limited number of iterations. Numerical results illustrate that: i) the proposed algorithm is an effective approach for obtaining near-optimal performance with acceptable complexity; and ii) the proposed NOMA-based D2D framework is capable of achieving promising gains over traditional orthogonal multiple access (OMA)-based D2D framework.
\end{abstract}

\section{INTRODUCTION}

Device-to-device (D2D) communications is considered as one of the pieces of the fifth generation $(5 \mathrm{G})$ jigsaw puzzle in order to improve spectral efficiency [1]. Driven by the potential benefits of $\mathrm{D} 2 \mathrm{D}$ communications, many works have been prompted recently under different scenarios [2-4]. Solution approaches that allowed cellular devices and D2D pairs to share spectrum resources were proposed in [2], thereby improved the spectral efficiency of traditional cellular networks. In [3], the functions to facilitate D2D session setup and mechanisms to control and limit the interference of D2D communications to the cellular network were illustrated. In [4], D2D spectrum sharing and mode selection using a hybrid network model and a unified analytical approach were jointly studied.

Apart from invoking D2D technique to improve the spectral efficiency of the wireless networks, another emerging technique-non-orthogonal multiple access (NOMA) - is able to address the spectral efficiency enhancement issue, on the standpoint of realizing a new power dimension for multiple access. Having been included in 3GPP long term evolution (LTE) [5], NOMA is regarded as one of the promising candidates in future $5 \mathrm{G}$ networks for its potential ability to significantly improve the spectral efficiency [6]. Different from the conventional orthogonal multiple access (OMA) technique, NOMA is capable of supporting users to share the same resource (e.g., time/frequency/code) with using different power level. In [7], a general downlink NOMA transmission scenario was considered in which one base station (BS) was capable of communicating with $M$ randomly deployed users. In [8], the fairness issue of NOMA networks was addressed with knowing different channel state information (CSI) at the BS. Considering the energy consumption issues, a new cooperative NOMA with invoking wireless power transfer protocol was proposed in [9].

Inspired by the aforementioned potential benefits of NOMA, we aim to investigate the promising application of NOMA technology in the D2D communications in this paper. More specifically, we propose a NOMA-based D2D communications framework. In this new framework, the proposed concept of "D2D group" means that one D2D transmitter is able to communicate with several D2D receivers via NOMA protocol, which is fundamentally different from the previous common used concept of "D2D pair". Several D2D groups are allowed to reuse the same subchannel occupied by a cellular user to improve the spectrum utilization. One of the common issues for both D2D and NOMA is the existence of interference among users in the same subchannel. As such, considering the interference management problem through investigating effective resource allocation strategies is more than necessary.

Note that allocating different D2D groups to different orthogonal subchannels generally turns out to be a non-deterministic polynomial-time (NP) hard problem. The computational prohibition with performing exhaustive search motivates us to invoke matching theory, which is a powerful tool for tackling this kind of combinatorial problems [10]. The main contributions of this work can be summarized as follows: 1) We formulate an optimization problem of resource allocation for the proposed NOMA-based D2D framework, aiming for maximizing the system sum rate; 2) With the aid of matching theory, we propose an effective resource allocation algorithm for obtaining a stable many-to-one matching between D2D groups and subchannels; 3 ) It is demonstrated that the proposed algorithm is capable of converging to a stable state within limited rounds of interaction and of achieving much lower complexity than a traditional combinational allocation; and 4) Numerical results demonstrate that the proposed algorithm can achieve the near-optimal system sum rate, and outperform the one-to-one matching algorithm. It is also shown that the NOMA-based D2D framework achieves higher system sum rate and larger number of accessed users than the OMA-based D2D framework.

\section{Network MODEL}

\section{A. System Description}

In this work, we focus on a single-cell uplink transmission scenario, as illustrated in Fig. 1(a). We consider that $M$ cellular users, i.e. $\mathcal{C}=\left\{C_{1}, \ldots, C_{M}\right\}$, communicate with one $\mathrm{BS}$ in the 
traditional cellular mode. Each cellular user $C_{m}$ is allocated in one subchannel $S C_{m} \in \mathcal{S C}, \mathcal{S C}=\left\{S C_{1}, \ldots, S C_{M}\right\}$ and all the subchannels are orthogonal with each other. There are $N$ D2D groups $\mathcal{D}=\left\{D_{1}, \ldots, D_{n}, \ldots, D_{N}\right\}$ communicating underlaying cellular networks. Unlike the traditional D2Dpair communications, we consider the $n$-th D2D transmitter $D T_{n}$ communicates with a group of $L_{n}$ D2D receivers, i.e., $\left\{D R_{1}, \ldots, D R_{k}, \ldots, D R_{L_{n}}\right\}$, simultaneously by applying NOMA transmission protocol, which introduces the concept of "D2D group" (as shown in Fig. 1(a)).

In Fig. 1(b), the interference received at each D2D receiver is illustrated. The intra-group interference refers to the interference of superposition signals from the D2D transmitter in the same D2D group. The inter-group interference indicates the interference from the D2D transmitters of other D2D groups that reuse the same subchannel. Last, the cellular interference represents the interference from the cellular user reusing the same subchannel.

It is assumed that the cellular users and D2D transmitters are uniformly distributed in the cell. The $L_{n}$ receivers in each D2D group are uniformly distributed within a disc with radius $d_{\max }$, and the origin of the disc is the corresponding $D T_{n}$. All channels are assumed to undergo quasi-static Rayleigh fading, where the channel coefficients are constant for each channel. We also assume that the perfect CSI is available at all D2D and cellular users.

\section{B. Channel Model}

We assume that each subchannel which is occupied by a cellular user can be reused by multiple D2D groups. As a consequence, the received signal at the BS corresponding to subchannel $S C_{m}$ is given by

$$
y_{m}=\sqrt{P_{c}} h_{m, b} x_{m}+\sum_{n} \eta_{n, m} \sqrt{P_{d}} g_{n, b} t_{n}+\zeta_{m},
$$

where $x_{m}$ and $t_{n}$ are the transmit signals of $C_{m}$ and $D T_{n}$, respectively. $\zeta_{m}$ is the additive white Gaussian noise (AWGN) at the BS on subchannel $S C_{m}$ with variance $\sigma^{2} . \eta_{n, m}$ represents the presence of interference, i.e., if $S C_{m}$ is assigned to $D_{n}$, $\eta_{n, m}=1$; otherwise, $\eta_{n, m}=0 . P_{c}$ and $P_{d}$ are the transmit power of the cellular users and D2D transmitters, respectively. In this paper, we assume that all the cellular users have the same transmit power and so do all the D2D transmitters. $h_{m, b}$ and $g_{n, b}$ are the channel coefficients including small-scale fading and path-loss between $C_{m}$ and the BS, and that between $D T_{n}$ and the BS, respectively.

Based on (1), we obtain the received signal-to-interferenceplus-noise ratio (SINR) at the BS corresponding to $S C_{m}$ as

$$
\gamma_{m}=\frac{P_{c}\left|h_{m, b}\right|^{2}}{\sum_{n} \eta_{n, m} P_{d}\left|g_{n, b}\right|^{2}+\sigma^{2}},
$$

where $\left|h_{m, b}\right|^{2}=\left|\hat{h}_{m, b}\right|^{2}\left(d_{1}^{m, b}\right)^{-\alpha}$ and $\left|g_{n, b}\right|^{2}=$ $\left|\hat{g}_{n, b}\right|^{2}\left(d_{2}^{n, b}\right)^{-\alpha}$. Here, $\hat{h}_{m, b}$ and $\hat{g}_{n, b}$ are small-scale fading with $\hat{h}_{m, b} \sim \mathcal{C N}(0,1)$ and $\hat{g}_{n, b} \sim \mathcal{C N}(0,1) . d_{1}^{m, b}$ is the distance from $C_{m}$ to the BS, and $d_{2}^{n, b}$ is the distance from $D T_{n}$ to the BS. $\alpha$ is the path-loss exponent.

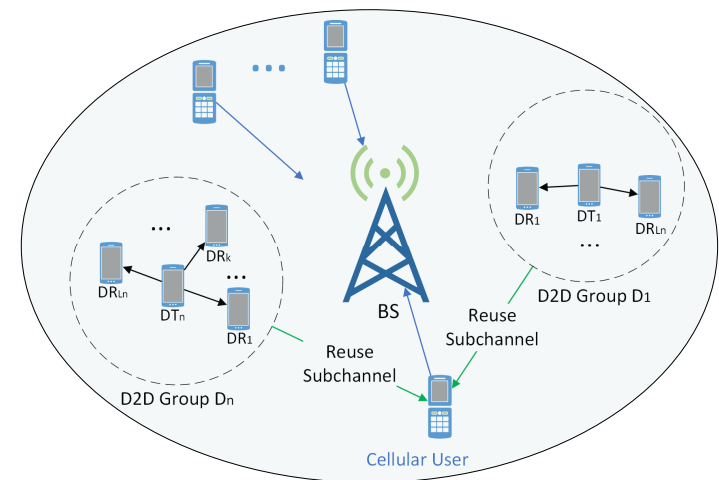

(a) Cellular layout

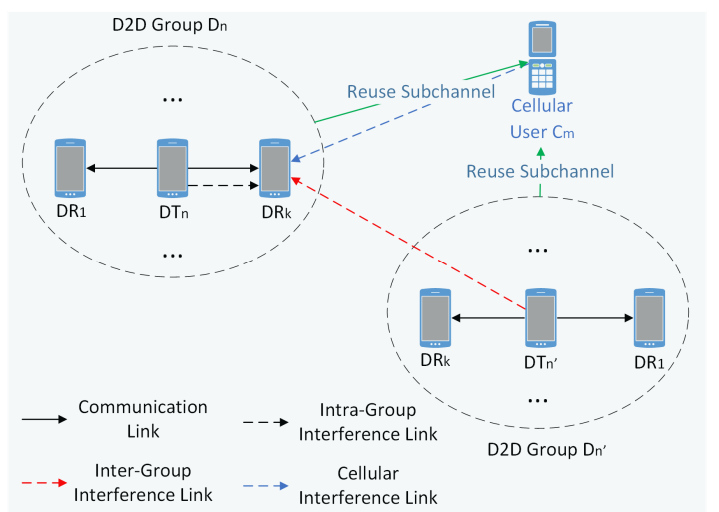

(b) Interference illustration

Fig. 1: Illustration of the NOMA-based D2D communications scenario.

The received signal at the $k$-th, $k \in\left\{1, \cdots, L_{n}\right\}$ receiver $D R_{n, k}$ in the $n$-th D2D group is given by

$$
\begin{aligned}
z_{n, k}= & f_{n, k} \sum_{k^{\prime}=1}^{L_{n}} \sqrt{a_{n, k^{\prime}} P_{d}} s_{n, k^{\prime}}+\sqrt{P_{c}} h_{m, n, k} x_{m} \\
& +\sum_{n * \neq n} \eta_{n *, n} \sqrt{P_{d}} g_{n *, n, k} t_{n *}+\zeta_{n, k},
\end{aligned}
$$

where $a_{n, k^{\prime}}$ denotes the power allocation coefficient for $D R_{n, k^{\prime}}$, and $s_{n, k^{\prime}}$ is the transmit signal from $D T_{n}$ to $D R_{n, k^{\prime}}$. $f_{n, k}, h_{m, n, k}$, and $g_{n *, n, k}$ are the channel coefficients between $D T_{n}$ and $D R_{n, k}$, that between $C_{m}$ and $D R_{n, k}$, and that between $D T_{n^{*}}$ and $D R_{n, k}$, respectively. $\zeta_{n, k}$ is the AWGN at $D R_{n, k}$ with variance $\sigma^{2} \cdot \eta_{n *, n}$ represents the presence of interference, i.e., if D2D group $D_{n}$ and $D_{n^{*}}$ reuse the same subchannel, $\eta_{n *, n}=1$; otherwise, $\eta_{n *, n}=0$.

For the NOMA-based D2D communications, without loss of generality, it is assumed that all channels in D2D group $D_{n}$ follows the order as $\left|f_{n, 1}\right|^{2} \leq\left|f_{n, 2}\right|^{2} \leq \cdots \leq\left|f_{n, L_{n}}\right|^{2}$. The power allocation coefficients of these NOMA users should follow the order as $a_{n, 1} \geq a_{n, 2} \geq \cdots \geq a_{n, L_{n}}$ with $\sum_{l=1}^{L_{n}} a_{n, l}=1$. As a result, successive interference cancellation (SIC) can be carried out at the users with stronger channels. Assuming that $1 \leq j \leq k<i$, the $k$-th user can decode the message of the $j$-th user and treats the message of the $i$-th user as interference. Specifically, the $k$-th user first decodes 
the messages of all the $(k-1)$ users with lower channel gains, and then successively subtracts these messages to obtain its own information. Therefore, according to the received signal expressed in (3), the received SINR at the $k$-th receiver in the $n$-th D2D group to decode the information of the $j$-th receiver is given by

$$
\gamma_{n, k}^{j}=\frac{\left|f_{n, k}\right|^{2} P_{d} a_{n, j}}{I_{n, k}^{\text {in }}+I_{n, k}^{\text {out }}+I_{n, k}^{c}+\sigma^{2}},
$$

where $I_{n, k}^{i n}=\left|f_{n, k}\right|^{2} P_{d} \sum_{i=j+1}^{L_{n}} a_{n, i}$ is the interference from the superimposed signals in the same D2D group, $I_{n, k}^{\text {out }}=\sum_{n * \neq n} \eta_{n *, n} P_{d}\left|g_{n *, n, k}\right|^{2}$ is the interference from other D2D groups, and $I_{n, k}^{c}=\sum_{m} \eta_{m, n} P_{c}\left|h_{m, n, k}\right|^{2}$ is the interference from the cellular user. Here, $\left|f_{n, k}\right|^{2}=$ $\left|\hat{f}_{n, k}\right|^{2}\left(d_{3}^{n, k}\right)^{-\alpha}, \quad\left|g_{n *, n, k}\right|^{2}=\left|\hat{g}_{n *, n, k}\right|^{2}\left(d_{5}^{n *, n}\right)^{-\alpha}$, and $\left|h_{m, n, k}\right|^{2}=\left|\hat{h}_{m, n, k}\right|^{2}\left(d_{4}^{m, n, k}\right)^{-\alpha}$. Note that the $L_{n}$-th receiver of the $n$-th D2D group can decode the signals of all the other receivers in the same group, thus the SINR is expressed as $\gamma_{n, L_{n}}^{L_{n}}=\frac{\left|f_{n, L_{n}}\right|^{2} P_{d} a_{n, L_{n}}}{I_{n, k}^{\text {out }}+I_{n, k}^{c}+\sigma^{2}}$. To ensure that the $k$-th user can decode the $j$-th user's signal, the following constraints should be satisfied: $\gamma_{n, k}^{j} \geq \gamma_{n, j}^{t h r}$ and $\gamma_{n, k}^{k} \geq \gamma_{n, k}^{t h r}, \forall k \in\left\{1, \ldots, L_{n}\right\}$. Here, $\gamma_{n, j}^{t h r}$ and $\gamma_{n, k}^{t h r}$ are the SINR thresholds for decoding the received signals of $D R_{j}$ and $D R_{k}$, respectively.

\section{Sum Rate}

Based on (2) and (4), the rate for the $m$-th cellular user $C_{m}$ and the sum rate for the $n$-th $\mathrm{D} 2 \mathrm{D}$ group $D_{n}$ are give by

$$
R_{m}=\log _{2}\left(1+\frac{P_{c}\left|h_{m, b}\right|^{2}}{\sum_{n} \eta_{n, m} P_{d}\left|g_{n, b}\right|^{2}+\sigma^{2}}\right)
$$

and

$$
\begin{aligned}
R_{n}= & \sum_{k=1}^{L_{n}-1} \log _{2}\left(1+\frac{\left|f_{n, k}\right|^{2} P_{d} a_{n, k}}{I_{n, k}^{\text {in }}+I_{n, k}^{\text {out }}+I_{n, k}^{c}+\sigma^{2}}\right) \\
& +\log _{2}\left(1+\frac{\left|f_{n, L_{n}}\right|^{2} P_{d} a_{n, L_{n}}}{I_{n, k}^{\text {out }}+I_{n, k}^{c}+\sigma^{2}}\right)
\end{aligned}
$$

respectively. As such, we can obtain the sum rate of all the cellular users and D2D groups as

$$
R_{\text {sum }}=\sum_{m=1}^{M}\left(R_{m}+\sum_{n=1}^{N} \eta_{n, m} R_{n}\right) .
$$

\section{PRoblem Formulation AND PRoposed OPTIMIZATION METHOD}

In this section, we formulate the problem to maximize the sum rate by allocating proper subchannels for the D2D groups. To solve the formulated problem, a novel algorithm based on the many-to-one two-sided matching is proposed. The theoretical analysis is provided to demonstrate the effectiveness of the proposed algorithm.

\section{A. Problem Formulation}

In order to maximize the sum rate given by (7), the optimization problem can be formulated as:

$$
\begin{gathered}
\max _{\eta_{n, m}} R_{\text {sum }}, \\
\text { s.t. } \quad \gamma_{n, k}^{k} \geq \gamma_{n, k}^{t h r}, \quad \forall n \in\{1, \ldots, N\}, k \in\left\{1, \ldots, L_{n}\right\},
\end{gathered}
$$

$$
\begin{gathered}
\gamma_{m} \geq \gamma_{m}^{t h r}, \quad \forall m \in\{1, \ldots, M\}, \\
\eta_{n, m} \in\{0,1\}, \quad \forall n \in\{1, \ldots, N\}, \forall m \in\{1, \ldots, M\}, \\
\sum_{m} \eta_{n, m} \leq 1, \quad \forall n \in\{1, \ldots, N\},
\end{gathered}
$$

where $\gamma_{n, k}^{t h r}$ and $\gamma_{m}^{t h r}$ are the SINR thresholds for the $k$-th receiver of the $n$-th D2D group and that for the $m$-th cellular user, respectively. The constraints in (8b) and (8c) represent that the SINR for D2D and cellular users should be larger than the threshold. Constraint (8d) shows that the value of $\eta_{n, m}$ should be either 0 or 1 . It is indicated in $(8 \mathrm{e})$ that at most one subchannel can be allocated to each D2D group.

The formulated problem is a mixed integer nonlinear programming (MINLP) problem [11], which is NP-hard to solve and exhaustive search yields prohibitive complexity. Therefore, we invoke the many-to-one two-sided matching theory for obtaining a suboptimal solution of the formulated problem.

\section{B. Proposed Subchannel Allocation Algorithm Based on Many- to-One Matching}

The matching problem we formulate here is the many-toone two sided matching between D2D groups and subchannels. The set of D2D groups and subchannels can be regarded as two opposite groups of selfish and rational players who try to enhance their own benefits during the matching process. To proceed with proposing the subchannel allocation algorithm, we first introduce some notations and basic definitions for the matching model.

Definition 1: In the many-to-one matching model, a matching $\Omega$ is a function from the set $\mathcal{S C} \cup \mathcal{D}$ into the set of all subsets of $\mathcal{S C} \cup \mathcal{D}$ such that 1) $\left|\Omega\left(D_{n}\right)\right|=1, \forall D_{n} \in \mathcal{D}$, and $\Omega\left(D_{n}\right)=$ $D_{n}$ if $\Omega\left(D_{n}\right) \notin \mathcal{S C}$; 2) $\left|\Omega\left(S C_{m}\right)\right| \leq N, \forall S C_{m} \in \mathcal{S C}$, and $\Omega\left(S C_{m}\right)=\emptyset$ if $S C_{m}$ is not matched to any D2D group; 3) $\Omega\left(D_{n}\right)=S C_{m}$ iff $D_{n} \in \Omega\left(S C_{m}\right)$.

D2D groups have preferences over individual subchannels, just as in a one-to-one matching model, and subchannels have preferences over subsets of $\mathcal{D}$. Thus, the preference list is given as: $\boldsymbol{P L}=\left\{\boldsymbol{P}\left(D_{1}\right), \ldots, \boldsymbol{P}\left(D_{N}\right), \boldsymbol{P}^{\dagger}\left(S C_{1}\right), \ldots, \boldsymbol{P}^{\dagger}\left(S C_{M}\right)\right\}$. Here, $\boldsymbol{P}\left(D_{n}\right)$ is the preference list of $D_{n}$ over the individual subchannels, and $\boldsymbol{P}^{\dagger}\left(S C_{m}\right)$ is the preference list of $S C_{m}$ over sets of D2D groups. The preference lists of players are formed in descending order with respect to the preference values. For the D2D groups, the preference value over each subchannel is the data rate of the D2D group. Thus, the relationship of "prefer" for D2D groups can be expressed as

$$
S C_{m} \succ_{D_{n}} S C_{m^{\prime}} \Leftrightarrow R_{n}^{m}>R_{n}^{m^{\prime}},
$$

where $R_{n}^{m}$ and $R_{n}^{m^{\prime}}$ are the data rates of $D_{n}$ when it reuses $S C_{m}$ and $S C_{m^{\prime}}$, respectively. $S C_{m} \succ_{D_{n}} S C_{m^{\prime}}$ implies that $D_{n}$ prefers $S C_{m}$ to $S C_{m^{\prime}}$.

Next we define the preference values for subchannels over a set of D2D groups. Although we allow multiple D2D groups to reuse the same subchannel, it is worth noticing that the sum rate on the latter term is deteriorated if the number of D2D groups occupying it keeps increasing. This is due to the fact that the co-channel interference enhances. Therefore, we define 
the preference values of subchannels on a set of D2D groups as the sum rate of all the D2D groups and the corresponding cellular user. If $S C_{m}$ prefers the set of D2D groups $\mathcal{S}$ to $\mathcal{S}^{\prime}$, the relationship between $\mathcal{S}$ and $\mathcal{S}^{\prime}$ can be expressed as

$$
\mathcal{S} \succ_{S C_{m}} \mathcal{S}^{\prime} \Leftrightarrow R_{m}^{\mathcal{S}}+\sum_{D_{n} \in \mathcal{S}} R_{n}^{m}>R_{m}^{\mathcal{S}^{\prime}}+\sum_{D_{n} \in \mathcal{S}^{\prime}} R_{n}^{m},
$$

where $R_{m}^{\mathcal{S}}$ and $R_{m}^{\mathcal{S}^{\prime}}$ are the data rates of $C_{m}$ sharing the subchannel with the set of D2D groups $\mathcal{S}$ and $\mathcal{S}^{\prime}$, respectively.

Faced with a set $\mathcal{S}$ of D2D groups, each subchannel $S C_{m}$ can determine which subset of $\mathcal{S}$ it would most prefer. Call this the choice of $S C_{m}$ from $\mathcal{S}$, and denote it by $C h_{S C_{m}}(\mathcal{S})$. That is, for any subset $\mathcal{S}$ of $\mathcal{D}$, the choice of $S C_{m}$ is $C h_{S C_{m}}(\mathcal{S})=\mathcal{S}^{\star}$ such that $\mathcal{S}^{\star} \subseteq \mathcal{S}$, and $\mathcal{S}^{\star} \succ_{S C_{m}} \mathcal{S}^{\star \star}$ for all $\mathcal{S}^{\star \star}$ contained in $\mathcal{S}$. The subchannels' preferences over sets of D2D groups have the property of substitutability, which is defined as follows.

Definition 2: The preference of a subchannel $S C_{m}$ over sets of D2D groups has the property of substitutability if, for any set $\mathcal{S}$ that contains D2D group $D_{n}$ and $D_{n^{\prime}}$, if $D_{n}$ is in $C h_{S C_{m}}(\mathcal{S})$, then $D_{n}$ is in $C h_{S C_{m}}\left(\mathcal{S} \backslash\left\{D_{n^{\prime}}\right\}\right)$.

A matching $\Omega$ can be improved upon by a pair $\left(D_{n}, S C_{m}\right)$ if $D_{n}$ and $S C_{m}$ are not matched at $\Omega$ but would both prefer if they are matched together, i.e. if $\Omega\left(D_{n}\right) \neq S C_{m}$ and if $S C_{m} \succ_{D_{n}} \Omega\left(D_{n}\right)$ and $D_{n} \in C h_{S C_{m}}\left(\Omega\left(S C_{m}\right) \cup\left\{D_{n}\right\}\right)$. In this case, we also call $\left(D_{n}, S C_{m}\right)$ a blocking pair. We define stable matching as the following.

Definition 3: A matching $\Omega$ is stable if it cannot be improved upon by any individual player or any pair $\left(D_{n}, S C_{m}\right)$.

Since the largest coalition it considers is a $\left(D_{n}, S C_{m}\right)$ pair, this is a definition of pairwise stability. It has been proved in [12] that, the set of stable matchings is always nonempty for matching models with substitutable preferences.

The work in [13] introduced an efficient algorithm, known as the deferred acceptance (DA) algorithm which can find a stable matching. Inspired by the DA algorithm, we propose a distributed matching algorithm, i.e. MTBSA, between D2D groups and subchannels, as shown in Algorithm 1. First, each D2D group $D_{n}, D_{n} \in \mathcal{D}$ forms a descending order preference list with respect to (9). Then each D2D group proposes to its most preferred subchannel, and each subchannel decides to accept or reject these proposals based on the preference values on the current set of D2D groups. In the subsequent steps, each D2D group which received one or more rejections at the previous steps proposes to its most preferred subchannel who has not yet rejected it. The algorithm stops after any step where all the D2D groups have been matched or the preference lists for all the unmatched D2D groups are empty.

\section{Analysis of The Proposed Algorithm}

To evaluate the effectiveness of the proposed algorithm, we analyze the properties of MTBSA in terms of stability, convergence and complexity in this subsection.

1) Stability: The final matching $\Omega^{*}$ of MTBSA is a pairwise-stable matching. The proof is given as follows:

Proof: In MTBSA, each D2D group proposes to its most preferred subchannel who has not previously rejected it. Assume

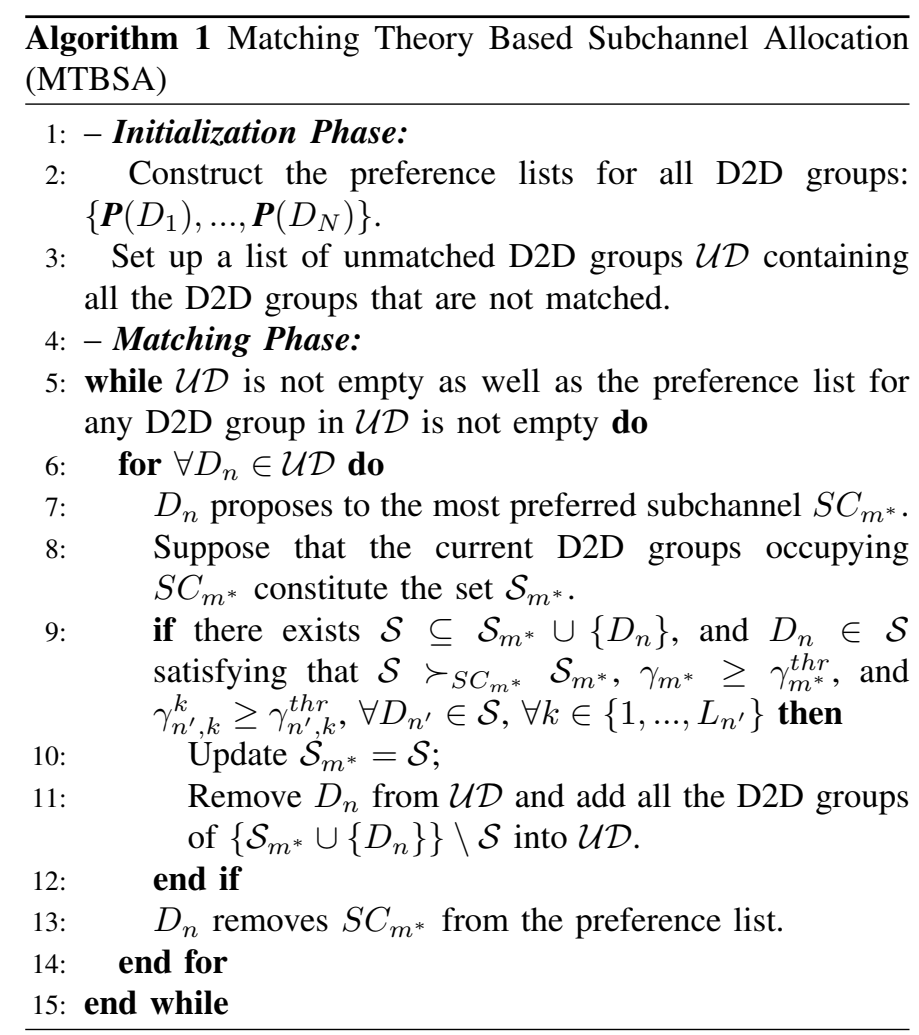

that there exists a blocking pair $\left(D_{n}, S C_{m}\right) \notin \Omega^{*}$ satisfying that 1) $S C_{m} \succ_{D_{n}} \Omega^{*}\left(D_{n}\right)$, and 2) $D_{n} \in C h_{S C_{m}}\left(\Omega^{*}\left(S C_{m}\right) \cup\right.$ $\left.\left\{D_{n}\right\}\right)$. At some step of the algorithm where the matching was $\Omega, D_{n}$ proposed to $S C_{m}$ and was subsequently rejected, so $D_{n}$ prefers $\Omega\left(D_{n}\right)$ to $S C_{m}$, and $\Omega$ is not improved upon by the pair $\left(D_{n}, S C_{m}\right)$. Since $D_{n}$ and $S C_{m}$ are arbitrary, $\Omega^{*}$ is pairwise stable.

2) Convergence: As shown in MTBSA, the matching process ends when all the D2D groups are matched or the preference lists for all unmatched D2D groups are empty. In each round, every unmatched D2D group proposes to its most preferred subchannel who has not previously rejected it. In other words, each D2D group proposes to each subchannel only once. After at most $M$ rounds, the preference lists for all D2D groups are empty, and no D2D group tends to make any further proposals even though it is not matched with any subchannel. Therefore, within limited number of rounds, the matching process converges to the final state which is stable.

3) Complexity: For the optimal approach, the exhaustive search needs to be performed on each subchannel. It is like that to partition an set with $1,2, \ldots, N$ elements into $M$ disjoint subsets with the sizes with $l_{1}^{n}, l_{2}^{n}, \ldots, l_{M}^{n}$, respectively, where the constraints $l_{1}^{n}+l_{2}^{n}+\ldots+l_{M}^{n}=n$ should be satisfied. Inspired by the multinomial theorem, the number of possible ways for allocating $n$ D2D groups to $M$ identical subchannels is given by $\left(\begin{array}{c}n \\ l_{1}+l_{2}+\ldots+l_{M}\end{array}\right)=n ! / \prod_{m=1}^{M} l_{m}^{n}$ !. Considering that all the subchannels are different in our scenario, the number of all the possible ways according to the exhaustive search can be expressed as $M ! \sum_{n=1}^{N} n ! / \prod_{m=1}^{M} l_{m}^{n} !$. Note that the complexity of the exhaustive search is mainly determined by 
the largest number $N$. As a consequence, we can obtain the approximate results as $\mathcal{O}\left(M ! N ! / \prod_{m=1}^{M} l_{m}^{N} !\right)$. For MTBSA, in the initialization phase, D2D groups need to set the preference lists, for which the complexity is $\mathcal{O}\left(N M^{2}\right)$. In the matching phase, each D2D group proposes at most $M$ times. Therefore, the total complexity of MTBSA is $\mathcal{O}\left(N M^{2}\right)$. Similarly, the complexity of the one-to-one matching algorithm is also $\mathcal{O}\left(N M^{2}\right)$. It is seen that the complexity of MTBSA is quite smaller than that of the exhaustive search when the number of D2D groups grows large. Table I summarizes the characteristics of the exhaustive search, the MTBSA, and the one-to-one matching algorithm, respectively. The comparison between the complexity and performance are clearly illustrated.

TABLE I: Comparison Among Different Algorithms

\begin{tabular}{|l|l|l|l|}
\hline Algorithm & Exhaustive & MTBSA & $\begin{array}{l}\text { One-to-one } \\
\text { matching }\end{array}$ \\
\hline Complexity & $\mathcal{O}\left(\frac{M ! N !}{\prod_{m=1}^{M} l_{m}^{N} !}\right)$ & $\mathcal{O}\left(N M^{2}\right)$ & $\mathcal{O}\left(N M^{2}\right)$ \\
\hline Total sum rate & Optimal & Near-optimal & Low \\
\hline $\begin{array}{l}\text { Number of accessed } \\
\text { D2D groups }\end{array}$ & High & High & Low \\
\hline
\end{tabular}

\section{Numerical Results AND Discussions}

In this section, numerical results are provided to demonstrate the performance of the proposed MTBSA algorithm. The specific parameters are summarized in Table II unless otherwise specified. The performance of the exhaustive search and the one-to-one matching based algorithm are provided as benchmarks for comparison to show the effectiveness of MTBSA. More particularly, the exhaustive search is capable of achieving the upper bound of the sum rate. In the one-to-one matching algorithm, one D2D group can use no more than one subchannel, and one subchannel can only be allocated to one D2D group. Additionally, the performance of the traditional OMA-based D2D communications is illustrated in an effort to demonstrate the potential benefits of the proposed NOMAbased D2D framework.

\section{TABLE II: Simulation Parameters}

\begin{tabular}{|l|l|}
\hline Cellular radius & $40 \mathrm{~m}$ \\
\hline Maximum distance between D2D pairs & $5 \mathrm{~m}$ \\
\hline Cellular-user SINR threshold & $1.8 \mathrm{~dB}$ \\
\hline D2D-user SINR threshold & $1.3 \mathrm{~dB}$ \\
\hline Transmission power of users & $23 \mathrm{dBm}$ \\
\hline Noise power & $-98 \mathrm{dBm}$ \\
\hline Path-loss exponent & 4 \\
\hline Number of subchannels & 3 \\
\hline
\end{tabular}

For simplicity, in the following simulation results, we assume that all the D2D groups have the same number of receivers, i.e., $L_{n}=K, \forall n \in\{1, \ldots, N\}$, and the power allocation for each receiver is fixed. In Fig. 2 to Fig. 4 , we set $K=3$ and the corresponding power allocation coefficients as $a_{1}=0.5$, $a_{2}=0.3$, and $a_{3}=0.2$. In Fig. 5, we give three values of $K$ : 1) $K=2$, where the power allocation coefficients are $a_{1}=$
0.6 , and $a_{2}=0.4$;2) $K=3$, where the power allocation coefficients are $a_{1}=0.5, a_{2}=0.3$, and $a_{3}=0.2$; and 3) $K=4$, where the power allocation coefficients are $a_{1}=0.4$, $a_{2}=0.3, a_{3}=0.2$, and $a_{4}=0.1$.

Fig. 2 shows that, the number of accessed D2D groups increases as the number of D2D groups in the network increases. This is because that with increasing $N$, the probability of D2D groups with less interference to the cellular UEs being assigned to them increases, which leads to the larger number of accessed D2D groups that can meet the SINR constraints of cellular UEs. This phenomenon is similar to the effect of multi-user diversity. It is worth noting that with the increase of the number of D2D groups in the network, the increasing rate of the number of accessed D2D groups becomes smaller due to the enhanced co-channel interference. One can also observe that the number of accessed D2D groups can get saturated quickly in the oneto-one matching algorithm. This is due to the fact that each subchannel can be allocated to no more than one D2D group.

Fig. 3 plots the total sum rate versus different number of D2D groups in the network. One can observe that the sum rate increases with the number of D2D groups, which follows the intuition that more D2D groups contribute to a higher total sum rate. It is also observed that MTBSA achieves much higher sum rate compared to the one-to-one matching algorithm. Meanwhile, MTBSA is capable of reaching around 95.8\% of the optimal result. Recall the complexity of the proposed algorithm, which is much lower than the exhaustive search, unequivocally substantiates the plausibility of the proposed algorithm. Fig. 3 also demonstrates that the NOMA-based D2D framework achieves larger sum rate than the traditional OMAbased D2D framework. It is worth pointing out that the power allocation among the D2D receivers is a key issue which will significantly influence the network performance for delivering higher sum rate. This problem is out of the scope of this paper and will be included in our future work.

Fig. 4 plots the number of accessed receivers versus different number of D2D groups in the network. It can be seen from the figure that the number of accessed receivers in the MTBSA is larger than that in the one-to-one matching algorithm. This is because more than one D2D groups are allowed to be allocated to one subchannel in the MTBSA, and thus the resource utilization is improved. It is also noted that the NOMA-based D2D communications achieves a larger number of accessed D2D receivers than the OMA-based D2D communications, which further shows the merits of applying NOMA transmission protocol in D2D communications.

Fig. 5 depicts the total sum rate versus different number of receivers in each D2D group. It can be seen that the sum rate increases as the number of receivers in each D2D group increases. However, if the number of receivers in each D2D group keeps increasing, the co-channel interference is enhanced, and thus the sum rate and number of accessed D2D groups may be deteriorated. Therefore, there exists an optimal value of the number of receivers in each D2D group, which can be a promising future research direction. 


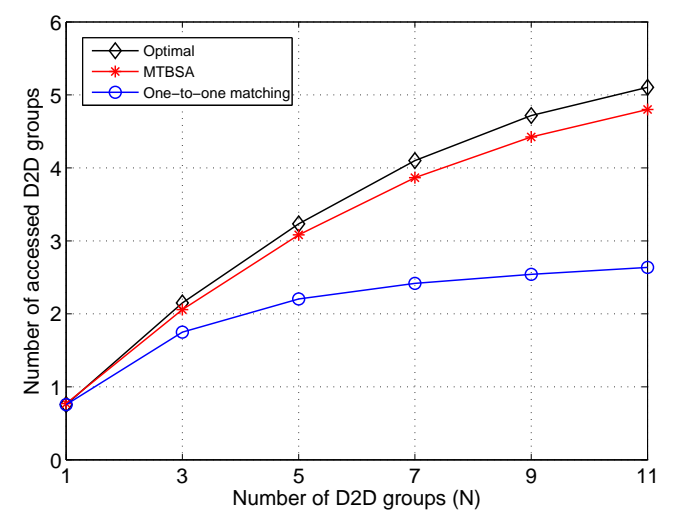

Fig. 2: Number of accessed D2D groups versus different number of $\mathrm{D} 2 \mathrm{D}$ groups in the network, with $\mathrm{K}=3$.

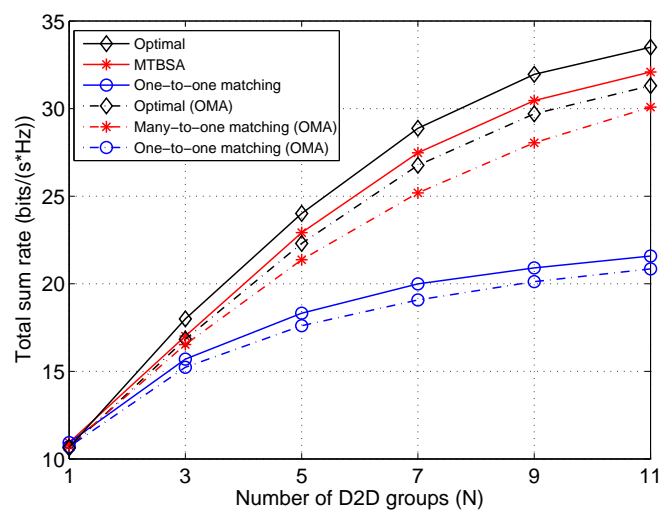

Fig. 3: Total sum rate versus different number of D2D groups in the network, with $\mathrm{K}=3$.

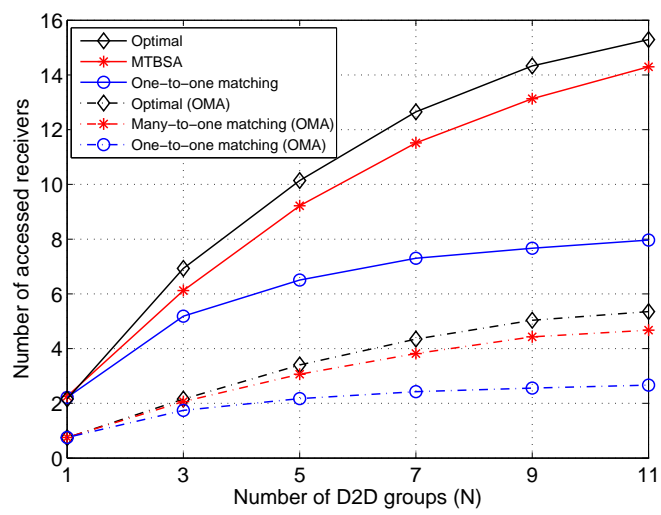

Fig. 4: Number of accessed receivers versus different number of $\mathrm{D} 2 \mathrm{D}$ groups in the network, with $\mathrm{K}=3$.

\section{CONCLUSION}

In this paper, the application of non-orthogonal multiple access (NOMA) to the device-to-device (D2D) communications has been studied. A novel algorithm invoking many-to-one matching theory has been proposed for tackling the subchan-

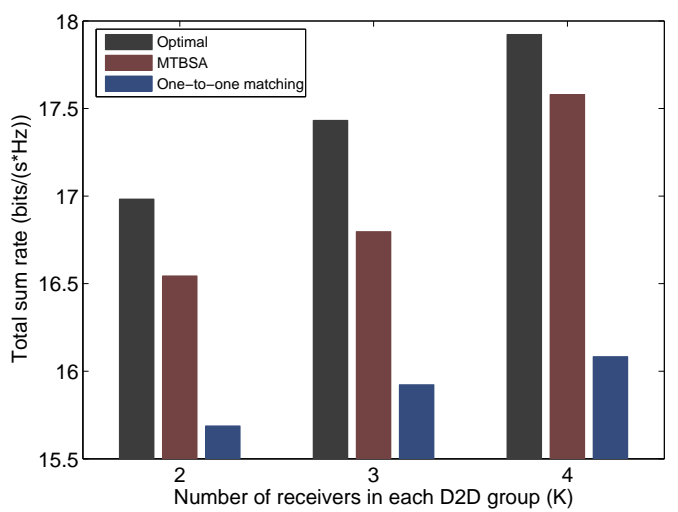

Fig. 5: Total sum rate versus different number of receivers in each D2D group, with $\mathrm{N}=3$.

nel allocation problem in the considered NOMA-based D2D framework. It was analytically demonstrated that the proposed algorithm had a acceptable complexity of $\mathcal{O}\left(N M^{2}\right)$, where a stable matching between the D2D groups and subchannels was obtained. Numerical results showed that the proposed algorithm achieved the near-optimal performance compared to the upper bound obtained by the exhaustive-search method. It was also shown that the proposed NOMA-based D2D framework outperformed the conventional OMA-based D2D framework.

\section{REFERENCES}

[1] J. G. Andrews and et al., "What will 5G be?" IEEE J. Sel. Areas Commun., vol. 32, no. 6, pp. 1065-1082, Jun. 2014

[2] G. Fodor and et al., "Design aspects of network assisted device-to-device communications," IEEE Commun. Mag., vol. 50, no. 3, pp. 170-177, Mar. 2012.

[3] K. Doppler, M. Rinne, C. Wijting, C. B. Ribeiro, and K. Hugl, "Device-todevice communication as an underlay to LTE-advanced networks," IEEE Commun. Mag., vol. 47, no. 12, pp. 42-49, Dec. 2009.

[4] X. Lin, J. G. Andrews, and A. Ghosh, "Spectrum sharing for deviceto-device communication in cellular networks," IEEE Trans. Wireless Commun., vol. 13, no. 12, pp. 6727-6740, Dec. 2014.

[5] Z. Ding and et al., "Application of non-orthogonal multiple access in LTE and 5G networks," IEEE Commun. Mag., submitted. [Online]. Available: http://arxiv.org/abs/1511.08610

[6] Y. Saito, A. Benjebbour, Y. Kishiyama, and T. Nakamura, "Systemlevel performance evaluation of downlink non-orthogonal multiple access (NOMA)," in Proc. IEEE Annual Symposium on Personal, Indoor and Mobile Radio Communications (PIMRC), London, UK, Sept. 2013.

[7] Z. Ding, Z. Yang, P. Fan, and H. V. Poor, "On the performance of nonorthogonal multiple access in 5G systems with randomly deployed users," IEEE Signal Process. Lett., vol. 21, no. 12, pp. 1501-1505, Dec. 2014.

[8] S. Timotheou and I. Krikidis, "Fairness for non-orthogonal multiple access in 5G systems," IEEE Signal Process. Lett., vol. 22, no. 10, pp. 1647-1651, Oct. 2015

[9] Y. Liu, Z. Ding, M. Elkashlan, and H. V. Poor, "Cooperative nonorthogonal multiple access with simultaneous wireless information and power transfer," IEEE J. Sel. Areas Commun., vol. 34, no. 4, Apr. 2016.

[10] Y. Gu, W. Saad, M. Bennis, M. Debbah, and Z. Han, "Matching theory for future wireless networks: fundamentals and applications," IEEE Commun. Mag., vol. 53, no. 5, pp. 52-59, May 2015.

[11] D. Bertsimas and J. N. Tsitsiklis, Introduction to linear optimization. Athena Scientific Belmont, MA, 1997, vol. 6

[12] A. E. Roth and M. A. O. Sotomayor, Two-sided matching: A study in game-theoretic modeling and analysis. Cambridge University Press, 1992, no. 18.

[13] D. Gale and L. S. Shapley, "College admissions and the stability of marriage," The American Mathematical Monthly, vol. 69, no. 1, pp. 9-15, Jan. 1962 